\title{
The evaluation of rapid serum tubes for various immunoassay tests
}

\author{
Esra Firat Oguz', (i) Emis Deniz Akbulut ${ }^{1}$, (i) Mujgan Ercan Karadag', (i) Canan Topcuoglu' ${ }^{\text {, }}$ (i) Turan Turhan \\ 'Department of Clinical Biochemistry, Ankara City Hospital, Ankara, Turkey \\ ${ }^{2}$ Department of Biochemistry, Harran University, Faculty of Medicine, Sanliurfa, Turkey
}

\begin{abstract}
Objectives: One of the most crucial steps on the preanalytical process that affect laboratory test results is proper and timely blood collection. In this study, we aimed to compare Rapid Serum Tubes (RST) and Serum Separator Tubes (SST) with no additive $Z$ tubes concerning various immunoassay tests.

Methods: Blood samples were collected from 50 healthy volunteers into three blood collection tubes. Sera from $Z$ tube, SST and RST were analysed simultaneously for thyroid-stimulating hormone (TSH), free triiodothyronine (fT3), free thyroxine (fT4), folate, vitamin B12, 25-OH vitamin D (25-OHD), parathyroid hormone (PTH), cortisol, ferritin, human chorionic gonadotropin (hCG) on DXI800 autoanalyser (Beckman Coulter, USA). The results were evaluated by comparing each tube pairs ( $Z$ tube-SST, Z tube-RST) according to desirable bias.

Results: There was no statistically significant difference between the two tube pairs for most analytes. There was not any significant clinical difference according to a desirable bias for the analytes tested.

Conclusion: RST offers acceptable clinical performance for the immunoassay tests performed in this study on DXI platform. The laboratory turn-around-times for immunoassay tests could be shortened approximately 15-20 minutes by the usage of RSTs.
\end{abstract}

Keywords: Preanalytical phase, rapid serum tube, Z tube

A ccurate results in medical laboratories play significant roles in the clinical decision-making process and also have influences on medical diagnoses and therapies. One of the most significant steps in preanalytical process that affect laboratory test results is proper and timely blood collection. However, the effects of blood collection tubes on laboratory test results are often overpassed [1]. Clinical Laboratory Standards Institute (CLSI) recommends performing analyses within two hours following blood collection to avoid prolonged contact of serum with cells and minimize errors in the preanalytical phase [2]. Preanalytical errors constitute $40-70 \%$ of total test errors [3-5]. Also, shorter turn-around-time (TAT) is significant for clinicians [6]. Most of the serum tubes used in clinical laboratories require a minimum 30 minutes of clotting time before centrifugation [2]. Using plasma specimens instead of serum to shorten the turnaround time of the test results can be an alternative choice [7]. However, there are some limitations of using plasma specimens as a different concentration of analytes and anticoagulants may reduce analyte stability. If serum is separated from the clot, it remains stable for a longer period [6].

Serum separator tubes and $Z$ tubes (with no additive) need a minimum of 30 minutes for clotting before centrifugation. Rapid serum tubes containing thrombin additive and provides a clotting time of five minutes to improve and shorten TAT.

In this study, we aimed to compare the immunoassay test parameters as follows: thyroid-stimulating hormone (TSH), free triiodothyronine (fT3), free thyroxine (fT4), folate, vita-

Address for correspondence: Esra Firat Oguz, MD. Department of Clinical Biochemistry, Ankara City Hospital, Ankara, Turkey Phone: +90 3125526000 E-mail: dr_esrafirat@hotmail.com ORCID: 0000-0002-8147-5379

Submitted Date: March 13, 2020 Accepted Date: June 16, 2020 Available Online Date: October 06, 2020

${ }^{\circ}$ Copyright 2020 by International Journal of Medical Biochemistry - Available online at www.internationalbiochemistry.com OPEN ACCESS This work is licensed under a Creative Commons Attribution-NonCommercial 4.0 International License. 
min $\mathrm{B} 12,25-\mathrm{OH}$ vitamin D (25-OHD), parathyroid hormone (PTH), cortisol, ferritin, human chorionic gonadotropin (hCG) in $B D$ vacutainer RST, BD vacutainer SST II and reference BD vacutainer $Z$ serum tubes.

\section{Materials and Methods}

\section{Subjects}

This study was conducted for two months period. Blood samples were collected from 50 apparently healthy volunteers for immunoassay tests. The subjects with any acute or chronic illness and taking medicine were excluded from this study. Informed consent was taken from each volunteer. This study was approved by the local Ethical Committee.

\section{Study design}

Blood samples were collected according to the Clinical Laboratory Standard Institute (CLSI) recommendations by an expert phlebotomist in the morning between 8 and 9 am after 12-hours fasting. The volunteers seated for 10 minutes before phlebotomy. Blood was collected using a standardized draw order according to CLSI document-GP41 [8].Tube 1: 7 $\mathrm{mL} B \mathrm{~B}$ vacutainer $\mathrm{Z}$ tube (Becton, Dickinson and Company Franklin Lakes, NJ, USA) contains no additive, no coating, Tube 2: $5 \mathrm{~mL}$ BD vacutainer serum separator tube II (SST) was coated with micronized silicone particles clot activator and contains gel barrier at the tube bottom, Tube 3: $5 \mathrm{~mL}$ BD vacutainer Rapid Serum Tube (RST) contains gel barrier polymer at the tube bottom and tube was coated with thrombin. Tubes were filled to capacity and gently inverted five or six times immediately after collection according to the manufacturer's recommendations. All of the tubes were left in an upright position at room temperature, RSTs were visually inspected for five minutes, $Z$ tubes and SSTs were allowed 30 minutes for clot formation. After clot formation, tubes were centrifuged at $1500 \times \mathrm{g}$ for 15 minutes according to the manufacturer's recommendations. Sera were separated, aliquoted and stored at $-80^{\circ} \mathrm{C}$ until analysis.

\section{Methods}

Sera from Z tube, SST II and RST were analysed randomly for thyroid-stimulating hormone (TSH), free triiodothyronine (fT3), free thyroxine (fT4), folate, vitamin B12, 25-OH vitamin D (25-OHD), parathyroid hormone (PTH), cortisol, ferritin, human chorionic gonadotropin (hCG) by paramagnetic particle, chemiluminescent immunoassay on DXI800 autoanalyser (Beckman Coulter, USA). All analyses were conducted in two level quality controlled the same run.

\section{Statistical analysis}

Statistical analysis was performed using the Statistical Package for Social Sciences (SPSS) program version 18.0 for Windows (SPSS Inc., Chicago, IL). The conformity of continuous variables to normal distribution was tested using the Kolmogorov-Smirnov test. The descriptive statistics of continuous variables were expressed as mean \pm standard deviation with normal distribution and median (min-max) with non-normal distribution. One way ANOVA test or Kruskal Wallis test was used to determine the presence of a statistically significant difference for three groups. If there was a statistically significant difference, Student's t-test for parametric and Mann Whitney-U test for non-parametric variables were performed for each paired tube groups. P-value $<0.05$ was considered statistically significant.

Bias was assessed using the formula: mean difference $(\%)=[$ (test tube mean - reference tube mean)/(reference tube mean)]x100 which should be less than desirable bias calculated from within-subject biological variation (CVw) and between-subject biological variation (CVG) (desirable bias $<0.25$ $\left.\left(\mathrm{CVw}^{2}+\mathrm{CV}_{\mathrm{G}}{ }^{2}\right)^{1 / 2}\right)$.

\section{Results}

Comparison of routine immunoassay test results between RST, SST and Z tubes for ten analytes is shown in Table 1. There was not any statistically significant difference for all analytes.

Table 1. Comparison of the results of three tubes (Z Tube-SST and RST)

\begin{tabular}{|c|c|c|c|c|c|c|}
\hline Analyte & Units & $\mathbf{n}$ & $Z$ tube & SST & RST & $\mathbf{p}$ \\
\hline T3 & $\mathrm{pg} / \mathrm{mL}$ & 50 & $3.07 \pm 0.33$ & $3.02 \pm 031$ & $2.96 \pm 0.30$ & 0.11 \\
\hline Folate & $\mathrm{ng} / \mathrm{mL}$ & 50 & $8.25 \pm .48$ & $8.24 \pm 3.40$ & $8.13 \pm 3.48$ & 0.92 \\
\hline Vitamin B12 & $\mathrm{pg} / \mathrm{mL}$ & 50 & $223(68-1061)$ & $219(61-1100)$ & $224(76-1194)$ & 0.88 \\
\hline Cortisol & $\mathrm{nmol} / \mathrm{L}$ & 50 & $10.05(5.10-23.27)$ & $10.12(4.71-23.18)$ & $10.71 \pm 3.46$ & 0.98 \\
\hline Ferritin & $\mu g / L$ & 50 & $28.1(2.40-241)$ & $26.7(2.60-250.5)$ & $28.1(2.30-251.10)$ & 0.86 \\
\hline hCG & $\mathrm{mIU} / \mathrm{mL}$ & 50 & $0.31(0.0-8.96)$ & $0.31(0.0-8.7)$ & $0.27(0.0-8.28)$ & 0.573 \\
\hline
\end{tabular}

SST: Serum separator tubes; RST: Rapid serum tubes 
We also compared the results of the analytes for tube pairs ( $Z$ tube-SST, $Z$ tube-RST) (Table 2). All of the calculated bias values of the analytes were below desirable bias.

\section{Discussion}

This study was designed to compare two different types of serum separator tubes (SST and RST) for the results of frequently performed immunoassay tests in the clinical laboratory. It was observed that the tubes showed no difference concerning TSH, fT4, folate, vitamin B12, 25-OH vitamin D, PTH and cortisol levels comparing with the reference tube ( $Z$ tube) on the Beckman Coulter DXI800 platform.

Turn-around time (TAT) is a significant quality indicator, particularly in the evaluation of emergency laboratory testing. Performing tests in plasma samples obtained from PSTs instead of using serum from standard serum tubes is a well-known strategy to reduce TATs. On the other hand, enough mixing of the specimen with anticoagulant immediately after blood drawing to ensure effective distribution of anticoagulant within the whole blood to prevent clotting is a very important and open to error step and emerges as a drawback of plasma over serum. The RSTs evaluated in the study are proposed to obtain serum samples faster than SSTs owing to shorter clotting time. As a result, reductions in TAT will improve the efficiency of laboratories and contribute to patient care. However, the effects of new equipment on test results should be evaluated before using in the routine clinical practice.

In a study conducted by Yan et al. RST was compared with the widely used serum separator tube (SST) for immunoassay tests on three common analyser platforms (Roche Modular, Abbott Architect, and Siemens Centaur analysers). A significant difference between the tubes according to both statistics and desirable bias (bias: $-15.3 \%, \mathrm{p}<0.01$ ) was observed for PTH on Abbott Architect System. According to the researchers, short half-life and reduced stability of serum PTH may be the reasons for the difference, and a detailed stability study on PTH for RST was suggested [9]. In our study, we also found no statistically significant difference and calculated bias was below the determined desirable bias limits. The difference in the results of the studies may be due to usage of the different types of kits for different analytical platforms, as antibodies from different origins (e.g., sheep and mouse) are used by manufacturers. In a study with Unical DxI platforms, comparing plasma separator tube (PST) and SST for many analytes, there was a significant difference concerning TSH between two tubes [10]. It is suggested that the difference may be related to the physiological difference between plasma and serum.

In the study of Yan et al., including 24 subjects, no significant difference between RST and SST for hCG on Roche Modular System was reported [9]. In the present study, including 50 subjects, the difference between RST and reference tube was statistically significant but without any accompanying significant clinical difference according to cut-off of hCG.

In our study, we used the $Z$ tube to exclude the effects of the additives. Also, all of the serum samples were transferred to the secondary tube, frozen, thawed and analysed.

Finally, data in this study show that RST and SST can provide comparable results with the $Z$ tube. There was not any significant clinical difference according to the desirable bias for the analytes tested.

One of the limitations of our study was that we only included healthy volunteers; we could not evaluate the performance of the tubes for pathological values. Also, we could not evaluate the stability of the tubes on different temperatures and time.

\section{Conclusion}

In a conclusion, RST offers acceptable clinical performance for the immunoassay tests performed in this study on the DXI

Table 2. Comparison of the tests for tube pairs ( $Z$ tube-SST and $Z$ tube-RST)

\begin{tabular}{|c|c|c|c|c|c|c|c|}
\hline Analyte & $\mathbf{n}$ & $Z$ tube & SST & RST & $\begin{array}{l}\text { Bias (\%) } \\
\text { (Z Tube- } \\
\text { SST) }\end{array}$ & $\begin{array}{l}\text { Bias (\%) } \\
\text { (Z Tube- } \\
\text { RST) }\end{array}$ & $\begin{array}{l}\text { Desirable } \\
\text { bias (\%) }\end{array}$ \\
\hline fT3 (pg/mL) & 50 & $3.07 \pm 0.33^{* *}$ & $3.02 \pm 031^{* *}$ & $2.96 \pm 0.30^{* *}$ & -1.62 & -3.58 & $4.8^{* * *}$ \\
\hline fT4 (ng/dL) & 50 & $0.89 \pm 0.13^{* *}$ & $0.88(0.68-3.56)^{*}$ & $0.88 \pm 0.13^{* *}$ & -1.12 & -1.12 & $3.3^{* * *}$ \\
\hline $250 \mathrm{OH}$ Vitamin D (ng/mL) & 50 & $13.15 \pm 6.05^{* *}$ & $13.3 \pm 6.23^{* *}$ & $13.28 \pm 6.34^{* *}$ & 1.14 & 0.98 & $10.5[12]$ \\
\hline PTH (pg/mL) & 50 & $46.4(9.40-165.6)^{*}$ & $46.5(9.20-179.7)^{*}$ & $45.9(9.20-173.7)^{*}$ & 0.21 & -1.07 & $8.8^{* * *}$ \\
\hline Cortisol (nmol/L) & 50 & $10.05(5.10-23.27)^{*}$ & $10.12(4.71-23.18)^{*}$ & $10.71 \pm 3.46^{* *}$ & 0.69 & 6.56 & $10.26^{* * *}$ \\
\hline Ferritin $(\mu \mathrm{g} / \mathrm{L})$ & 50 & $28.1(2.40-241)^{*}$ & $26.7(2.60-250.5)^{*}$ & $28.1(2.30-251.10)^{*}$ & -4.98 & 0.36 & $5.2^{* * *}$ \\
\hline$\beta h C G(\mathrm{mlU} / \mathrm{mL})$ & 50 & $0.31(0.0-8.96)^{*}$ & $0.31(0.0-8.7)^{*}$ & $0.27(0.0-8.28)^{*}$ & 1.69 & -11.47 & - \\
\hline
\end{tabular}

*: median (min-max). ${ }^{* *}$ : mean \pm SD. ${ }^{* * *}$ : desirable bias data is based on https://www.westgard.com/biodatabase1.htm (exception of $250 H$ vitamin D and vitamin B12). Z Tube: 7 $\mathrm{mL}$ BD Vacutainer Z; SST: $5 \mathrm{~mL}$ BD Vacutainer SST II advance; RST: $5 \mathrm{~mL}$ BD vacutainer Rapid Serum Tube 
platform. The laboratory turns around time for immunoassay tests could be shortened approximately 15-20 minutes using RSTs. Further studies with a larger number of tests and samples are needed to provide more relevant information.

Conflict of Interest: None declared.

Ethics Committee Approval: This study was approved by the local Ethical Committee.

Financial Disclosure: The authors declared that this study has received no financial support.

Peer-review: Externally peer-reviewed.

Authorship Contributions: Concept - E.F.O., E.D.A., M.E.K., C.T., T.T.; Design - E.F.O., E.D.A., M.E.K.; Supervision - E.F.O., E.D.A., M.E.K.; Funding - None; Materials - E.F.O., E.D.A., M.E.K., C.T., T.T.; Data collection \&/or processing - E.F.O., E.D.A., M.E.K.; Analysis and/or interpretation - E.F.O., E.D.A., M.E..K, C.T., T.T.; Literature search - E.F.O., E.D.A., M.E.K.; Writing - E.F.O., E.D.A., M.E.K.; Critical review - E.F.O., E.D.A., M.E.K., C.T., T.T.

\section{References}

1. Bowen RA, Remaley AT. Interferences from blood collection tube components on clinical chemistry assays. Biochem Med (Zagreb) 2014;24(1):31-44. [CrossRef]

2. Kiechle FL, Betsou F, Blakeney J, Calam RR, Catalasan IM, Raj P, et al. Procedures for the handling and processing of blood specimens for common laboratory tests. Approved Guideline. Clinical Laboratory and Standards Institue 2010;30(10):H18-A4.

3. Bonini P, Plebani M, Ceriotti F, Rubboli F. Errors in laboratory medicine. Clin Chem 2002;48(5):691-8. [CrossRef]
4. Carraro P, Plebani M. Errors in a stat laboratory: types and frequencies 10 years later. Clin Chem 2007;53(7):1338-42. [CrossRef]

5. Plebani M, Sciacovelli L, Aita A, Padoan A, Chiozza ML. Quality indicators to detect pre-analytical errors in laboratory testing. Clin Chim Acta 2014;432:44-8. [CrossRef]

6. Kocijancic M, Cargonja J, Delic-Knezevic A. Evaluation of the BD Vacutainer $\left.{ }^{\circledR}\right)$ RST blood collection tube for routine chemistry analytes: clinical significance of differences and stability study. Biochem Med (Zagreb) 2014;24(3):368-75. [CrossRef]

7. Chance J, Berube J, Vandersmissen M, Blanckaert N. Evaluation of the BD Vacutainer PST II blood collection tube for special chemistry analytes. Clin Chem Lab Med 2009;47(3):358-61.

8. CLSI. Collection of Diagnostic Venous Blood Specimens. 7th ed. CLSI standard GP41. Wayne, PA: Clinical and Laboratory Standards Institute; 2017.

9. Yan R, Lou A, Watts G, Tarr H, Smith H, Kinney L, et al. Comparison of Becton Dickinson Vacutainer rapid serum tube with the serum separator tube for routine chemistry and immunoassay tests. J Clin Pathol 2014;67(7):599-604. [CrossRef]

10. Strathmann FG, Ka MM, Rainey PM, Baird GS. Use of the BD vacutainer rapid serum tube reduces false-positive results for selected beckman coulter Unicel Dxl immunoassays. Am J Clin Pathol 2011;136(2):325-9. [CrossRef]

11. Kristensen GB, Rustad P, Berg JP, Aakre KM. Analytical Bias Exceeding Desirable Quality Goal in 4 out of 5 Common Immunoassays: Results of a Native Single Serum Sample External Quality Assessment Program for Cobalamin, Folate, Ferritin, Thyroid-Stimulating Hormone, and Free T4 Analyses. Clin Chem 2016;62(9):1255-63. [CrossRef]

12. Viljoen A, Singh DK, Farrington K, Twomey PJ. Analytical quality goals for 25-vitamin $D$ based on biological variation. J Clin Lab Anal 2011;25(2):130-3. [CrossRef] 